Stefano Triberti, Serena Barello, Guendalina Graffigna, Giuseppe

Riva, Antonio Candelieri, Francesco Archetti

\title{
Evaluating Patient Engagement and User Experience of a Positive Technology Intervention: The H-CIM Case
}

\begin{abstract}
The present chapter will provide an example of an intervention evaluation from the joint viewpoints of patient engagement and user experience. The authors evaluated H-CIM, a technological platform for the intelligent monitoring of physiological data of elderly patients performing physiotherapy exercises. Descriptive quantitative measures, behavioral observation, and qualitative interviews are integrated to evaluate H-CIM ability in (1) guaranteeing a positive experience to its users and (2) supporting them in advancing through a patient engagement development. This contribution would constitute a practical example of how these fundamental factors should be considered and evaluated when implementing positive technology for healthcare.
\end{abstract}

Keywords: Rehabilitation; Positive Technologies; User Experience; Patient Engagement; Intelligent Monitoring.

\section{Technologies for Patient Engagement: a Roadmap of Methodologies to Develop Effective e-Health Interventions}

In this chapter the authors will discuss how the PHE model could be useful to orientate the evaluation of a technology designed to augment the patient's clinical experience and health outcomes. The discipline of user experience and the patient engagement theoretical perspective may be insightful paradigms to be integrated. The authors shall discuss how these perspectives may be jointly useful to developing methodological guidelines in order to design and evaluate technologies for health which are in line with the patient's care needs. As described in Chapters 1 and 4:

- Patient engagement is a crucial objective to be pursued in the context of healthcare interventions, above all where patients are asked to face long term care interventions;

- New technologies, according to the positive technologies paradigm, constitute a fundamental resource to achieving patient engagement as they may improve the effect of clinical interventions by fostering patient adherence to treatments and

(c) BY-NC-ND $\odot 2015$ Stefano Triberti, Serena Barello, Guendalina Graffigna, Giuseppe Riva, Antonio Candelieri, 
ease the transfer of medical prescriptions from the hospital settings to the home and the everyday life.

In order to demonstrate the value of integrating the patient engagement and user experience perspectives for developing effective technology-mediated care plans, we will provide an illustrative case based on a technology designed for rehabilitative interventions.

\section{The Intervention Field: New Technologies for the Physical Rehabilitation of the Elderly}

Considering the societal challenges described in Chapter 1, healthcare services aimed at providing care to chronic elderly populations are devoting themselves to the search for new ways to ensure effective and efficient treatment programs to augment health outcomes in the long term by actively engaging health consumers (Laver, Ratcliffe, George, Burgess, \& Crotty, 2011;Barello, Graffigna, \& Vegni, 2012; Gruman et al., 2010; Graffigna et al., 2014). In particular, the physiotherapy rehabilitation field constitutes a relevant setting to exemplify the relevance of developing health technologies aimed at sustaining patient engagement in the care process.

Rehabilitation, on the one hand, assists patients who have sustained damage to the motor system as a result of acute clinical events, for example strokes (Jette et al., 2005); also, it allows the patient to improve damaged motor skills (even in cases of severe disability) related to the physiological aging process (Persad, Cook, \& Giordani, 2010). In both cases, the engagement of patients is essential to reducing the disability resulting from the disease and to support the recovery of an active role in the society. Many of the new methods concerning the use of positive technologies that are currently being investigated are already demonstrating encouraging results in supporting the process of treatment and rehabilitation (Graffigna, Barello, Wiederhold, Bosio, \& Riva, 2013; Riva, Baños, Botella, Wiederhold, \& Gaggioli, 2012; Rizzo \& Kim, 2005). Regarding physical rehabilitation and physiotherapy, the following technologies are widely used:

- Video games (usually based on analogical devices such as the Nintendo Wii) or virtual reality (Laver et al., 2011; Maggiorini, Ripamonti, \& Zanon, 2012; Smith \& Schoene, 2012)

- $\quad$ Robotics to support movement (assistive exoskeletons) (Forlizzi, DiSalvo, \& Gemperle, 2004; Kousidou, Tsagarakis, Caldwell, \& Smith, 2006; Lund, 2009)

- Tele-rehabilitation, or multimedia communication between patients and therapists (Tousignant et al., 2011) 
As suggested by the aforementioned studies, these profoundly heterogeneous technologies share some potential to support the rehabilitation process:

- When compared with "traditional” rehabilitation methods (not supported by technology) rehabilitation involving technological aspects is equally effective with respect to the goals of rehabilitation (Scherer, 2002), ensuring the automation of the exercises and the autonomy of the patient (Huete, Victores, Martinez, Gimenez, \& Balaguer, 2012) and consequently reducing the care costs (Fuhrer, 2007).

- Moreover, patients evaluate them as interesting, fun, and engaging, and their positive attitude is often linked to the overall success of the care experience (Kintsch \& DePaula, 2002)

New technologies are characterized by the ability to structure, augment, or replace the patient's experience, promoting strengthening of capacity, but above all a positive and self-fulfilling experience (Riva et al., 2012).

However, the outcome of the rehabilitation process does not directly emerge from the use of a health technology. Indeed, it is not sufficient that the technology "works well" from a technical viewpoint. Moreover, the patient must be able to use it and feel actively engaged in the experience of the intervention.

\section{Developing Health Technologies for Patient Enga- gement: a Methodological Road Map Based on the PHE Model}

As health technologies are becoming more affordable and acceptable, this is an opportune time to widely adopt them to better engage patients in the self-management of chronic diseases. Thus, adopting the right technology should be a priority for healthcare systems devoted to sustaining long term engagement of health consumers. To this aim, there is the need to make use of methodologies aimed at designing and evaluating technologies in order to really promote patient engagement and demonstrate its effectiveness at the clinical level.

As just discussed in Chapter 2, the PHE model demonstrates how patient engagement gradually develops along the medical course. Moreover, each phase of the engagement process features specific care needs and priorities. In order to promote effective technology-mediated care plans, it is fundamental to map the patient engagement experience and to align the care interventions to it.

So often, the development of health technologies risks leaving the human element and context of use behind. However, we can only generate progress towards the crucial goal of patient engagement for health if we focus on peoples' care experience. People are emotional and individuals are networked, whereas today's health- 
care systems are stuck on transaction-based models that don't meet people where they are, this way not being capable of anticipating their future care needs.

In the light of the PHE model, health technologies built by analyzing the user experience may sustain engagement by truly understanding the individuals' care expectations. The in-depth analysis of the user experience allows the understanding of patients' behaviour, motivations, and goals, and to use these to craft desirable experiences that enhance their interaction with health technologies and amplify their effect on clinical outcomes.

Patient engagement interventions need to begin with clearly understanding the specific human qualities of the patients we aim to serve. Technologies which are in line with these principles may be more effective than others not taking into account the patients' mindset concerning their health status.

To analyse the user experience in the case of healthcare, means to understand the patients' experience of interaction with technologies designed to sustain their clinical process. This experience features multifaceted dynamics and subjective reactions that may hinder or foster the effectiveness of the technologies themselves in promoting patient engagement.

Due to the variegated nuances of the patient care experience, multiple inquiry tools may be used to understand its different aspects. In depth interviews, questionnaires/self-reports, and observations of patients during or after the interaction with the technology, may constitute a useful toolbox to enable the development and evaluation of user-centered technologies really attuned with the engagement phase the patient is passing through. These methods, if combined, may produce a detailed picture of fundamental aspects of the patients' care process - i.e. illness experience, care expectations, wellness plans, and meanings associated with health technologies - which are all useful to designing technological instruments for patient engagement (see Figure 1).

These tools may be useful to:

- Evaluate the user experience with the technology

- Evaluate the technology's abilities in promoting and sustaining patient engagement

\section{Sustaining Patient Engagement in Rehabilitation Activities: a Case Study of the H-CIM Platform}

Let us now see how these research methods for understanding user experience may be used to analyze the effectiveness of a technological platform designed to promote patient engagement in rehabilitation activities. This case could be informative for technology developers and clinicians when developing and applying technologies in the care process and to ensure that they are able to sustain patient engagement in the care process. 


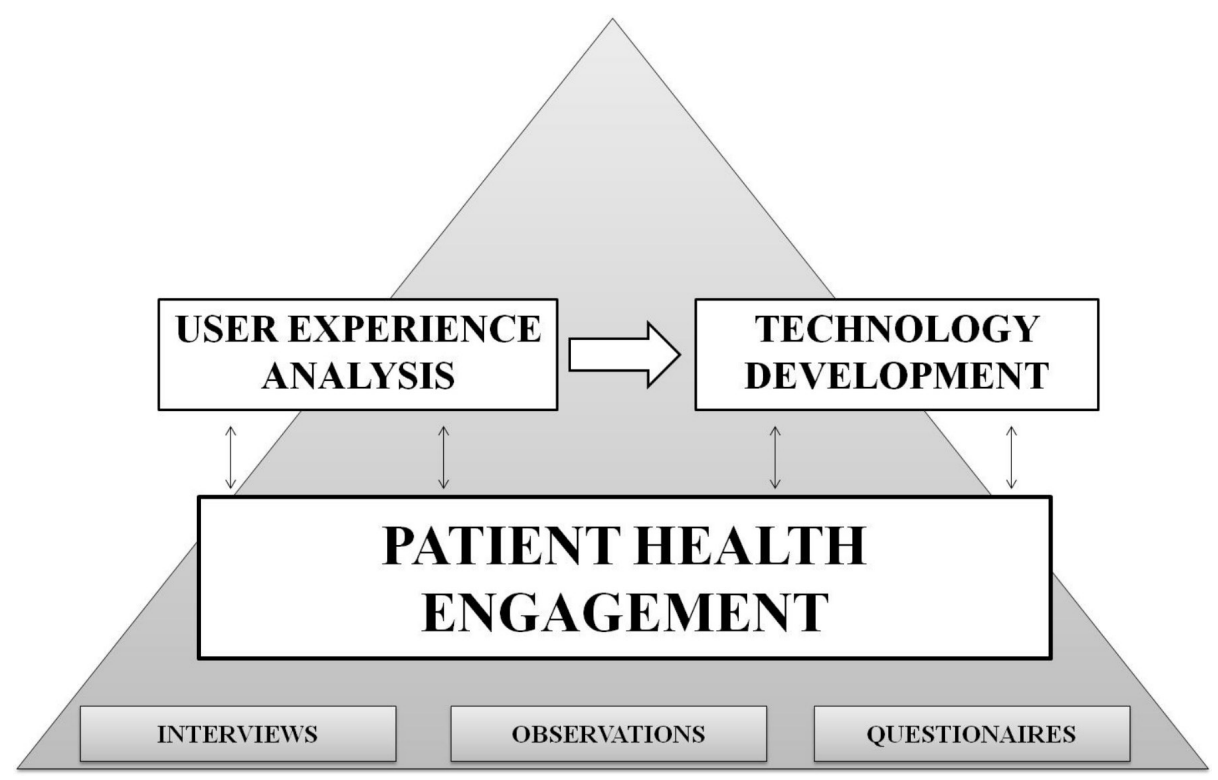

Figure 1: User experience analysis of e-Health technologies for patient engagement: a methodological framework.

$\mathrm{H}$-CIM (Health-Care Intelligent Monitoring) is a platform that detects physiological signals regarding the state of health and activity of an elderly patient at home, acting in his/her daily living environment. The "home processor", this way labelled because it is a personal computer placed in the house of the patient, records by three sources of data:

- Environment (network of ambient sensors). These are related to environmental parameters and to the presence of subjects within the frame of reference;

- Movement (wearable). These data are acquired through a set of devices worn by the monitored subject (accelerations of the dominant upper limb, trunk, and lower limb with respect to the three axes);

- Bio-signals (wearable). These data are acquired through a device worn by the patient and refer to the parameters of medical-clinical interest (e.g., heart rate) to be monitored during the various activities conducted by the patient.

This technology has been used to monitor the patients when they were performing rehabilitation exercises: the elderly patient should be able to perform the exercises autonomously, enabling the recording of the signals, and thus giving immediate information about his/her current process to the physician. This allows the physician to monitor the status of the patient in his/her daily life (1) and in the context of exercises that are a part of his/her rehabilitation (2).

The authors observed the test sessions of the technology which involved three elderly patients. The patients performed rehabilitation exercises shown by the phys- 


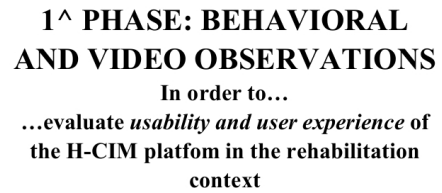

1^ PHASE: BEHAVIORAL AND VIDEO OBSERVATIONS In order to... ...evaluate usability and user experience of the H-CIM platfom in the rehabilitation context

$2^{\wedge}$ PHASE: SEMI-STRUCTURED

INTERVIEWS

In order to...

...understand how the H-CIM technology can

promote the engagement of the patients in their own care process

Figure 2: Main of the user experience analysis.

iotherapist in a video, while wearable and environmental sensors registered their physiological parameters.

\subsection{User Experience Evaluation}

This case study (three prototypical subjects, mean age 72 years, stable clinical condition) has provided two research modules covering respectively the first and second aims (see Figure 2). In the first phase, behavioural observations were designed to track the "in-vivo" interaction modalities between the patient and the technology when they were asked to complete physiotherapy exercises. Semi-structured interviews were then administered to the subjects, with the aim of understanding the experience of interacting with technology in order to comprehend how it can facilitate their engagement in the care process. We assessed the patients' engagement position by asking four questions (see Chapter 2 for more details about the instrument), which evaluate the patients' psychological attitude toward their health condition management. This set of questions - and the corresponding responses that the model interprets- allowed us to also assess the patients' disposition toward interacting with technologies for self-care. We assessed the state of health literacy of the patient (his/ her ability to think about the disease and its management by using the e-health technology); the state of emotional elaboration about the diagnosis and the health condition (his/her way of "feeling" when reflecting on his/her health status and on the use of the technology); and the consequent behavioural competences related to the self management of his/her own care (his/her "acting" attitude towards the disease and the use of the technological platform).

This multi-technique method (Denzin \& Lincoln, 2000) allowed for the detection of multifaceted data to obtain a composite view of the implemented technology and of its potential benefits for the rehabilitation process.

The patients also filled in a number of self-report measures in order to provide descriptive data about their own experience with the technology. More specifically, mental workload (Hart \& Staveland, 1988), system usability (Brooke, 1996), and possible negative emotional responses (Spielberger, Gorsuch, Lushene, Vagg, \& Jacobs, 1983) were assessed. Furthermore, the Patient Activation Measure (Hibbard, Stockard, Mahoney, \& Tusler, 2004) was employed in order to assess important aspects of 
patient engagement. Figure 3 illustrates the main outcomes of the user experience analysis and shows which aspects need to be considered in order to evaluate the effectiveness of the platform for medical treatment.

\subsection{What Are the Implications for Patient Engagement?}

Generally speaking, observations, interviews, and self-report showed that the H-CIM platform allows for the efficient tele-monitoring of patients' status and the physiological effects of exercise rehabilitation. Furthermore, HCIM has a positive impact on the patient care experience, thus promoting the enhancement of their health engagement. We will now examine in more detail some clinical cases that exemplify the patient interaction with technology according to the PHE model.

Across the clinical cases a positive attitude towards the technology was observed. However, the patient engagement position (according to the PHE model) orientates the patient's ways of interaction with the platform and the patient's disposition to utilize it in the long term.

Patient case 1: Maria, 68 years old - phase of arousal

Maria has been prescribed physical rehabilitation after a diagnosis of osteoporosis. She is worried about the consequences of this diagnosis for her health (feel) and she doesn't feel confident with the information the physician gave her about disease management (think). She generally feels a lack of skills to autonomously manage the medical regimen (act). In this framework, the patient mindset about her availability to use the technological platform is low. Unless the patient shows a positive attitude towards the technology, she has not fully its purpose, nor the way in which she could use it in her daily life.

“... I was only there and I just did the exercises but I have no idea, I am totally incompetent....... they are investigating many things no? Reactions, the reaction rate, but in the end I cannot understand because they did not tell me ..."

Patient case 2: Giovanna, 72 years old - phase of adhesion

Giovanna has been involved in a rehabilitation program after a meniscus injury. She feels confident concerning the rationale behind the rehabilitative prescriptions (think) and aware is of the disease implications for her life (feel). She describes herself as highly effective when managing her symptoms and the disease (act) and is satisfied with the relationship with her doctors. However, the patient shows some difficulties when she has to maintain the life style changes in the long term.

This makes the continuative use of the platform difficult if administered at home without clinical supervision. She needs to be monitored and still supported in effectively using it when alone. 

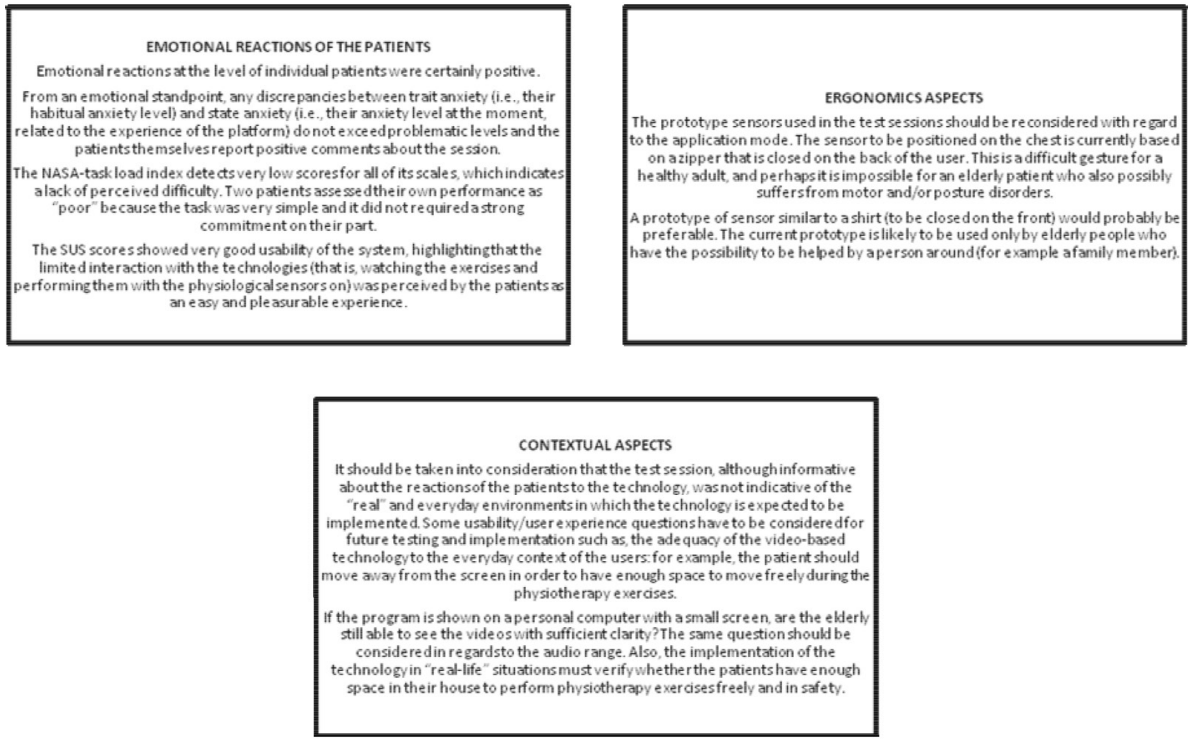

Figure 3: The multi-technique research design.

"I can't imagine using the platform all days. If I am alone I do not feel motivated to complete my exercises...”

\section{Patient case 3: Anna, 66 years old - phase of eudaimonic project}

Anna has been attending the rehabilitation program for one year after being diagnosed with lumbosciatica some years earlier. The patient is confident (feel) and resilient in her health management (think), even when troubles with therapeutic prescriptions emerge. She is also able to ask her physician for help on demand (act). She has integrated the use of the technological platform in her daily life and she perceives it as a means to having control over her body signals.

"The experience with technology is super positive. I feel protected, perhaps because there was a doctor and the physical therapist ... it's as if they are always there. In think that technologies can be useful, they are definitely an incentive to be more active in your health management"

The concept of technology is now available and present in the minds of the patients, who understand the value and the potential associated with the use of tools such as computers, smartphones, and tablets, but also other tools for monitoring the clinical condition in everyday life (e.g., holter, remote alarm). The perception of technology is positive. Indeed, the technology is perceived as a means by which patients feel monitored and followed up by the physician, who can control the parameters during the execution of the rehabilitation exercises.

Patients also perceive the technology as a means of amplifying the attention of the doctor towards them, promoting care continuity. This also makes them more 
secure and confident while performing the rehabilitation exercises, because they feel that they are in a safe environment. Finally, patients recognize the value of therapeutic prescriptions being transferred from the hospital setting to the home.

In general, the patients' testimonies reveal the value of being personally involved in the management of their own health, gradually acquiring information and tools provided by professionals and adapting them to their everyday lives.

Health appears to be a theme emerging from the reports of patients who, also in connection with the phase of life in which they find themselves, feel the need to be able to face their own life issues by maintaining a healthy and active lifestyle. Patients report a positive attitude with respect to the use of technology to support the rehabilitation process, provided it does not limit them in their daily lives.

For these reasons, the the technology designed to support the rehabilitation must take account of these needs expressed, as it is considered useful only if it does not imply renunciation and constraints for the user. What mainly hinders the involvement of patients in the use of technology seems to be related to the absence of clear information about the rationale behind the use of the platform and about its value compared to traditional rehabilitation methods. In addition, the technology is perceived by patients as possibly making them "passive”, not-autonomous.

This case provides clear indications for enhancing the experience and effectiveness of the H-CIM technology in sustaining patient engagement.

The experience with H-CIM appears to be related to positive sensations and emotions of the patients. The patients are clearly compliant with the interaction possibilities and with the affordances the technology offers to perform their rehabilitation exercises in an innovative way. In general, this positive attitude is an encouraging cue about the H-CIM technology constituting an effective tool in order to engage the patients in a specific aspect of their own care process.

On this basis, the H-CIM intervention can be analyzed in the light of the Patient Engagement Model (see Chapter 2 in this book). Using H-CIM technology, the patients are accompanied through the phase of adhesion since they positively and actively adhere to the rehabilitation prescription and also become more curious about their own therapy as it is administered via a new communication technology. At the same time, they raise concerns about how to effectively manage the technology use in the context of their everyday life, which consists of daily needs, tasks, and opportunities. Moreover, despite the positive feelings they have, they often ask for further explanations about how the technology works and why exactly is it useful for their health. In this sense, H-CIM can position itself at the third phase of the patient engagement process (see Figure 4), which is a very desirable position for a technological product at its first stages of testing. Moreover, in order to become an excellent tool for engaging patients in their own care process, H-CIM has a goal of reaching the eudaimonic project phase (see Figure 4), that is, it has to be effectively included in the patients' daily life and their needs. 


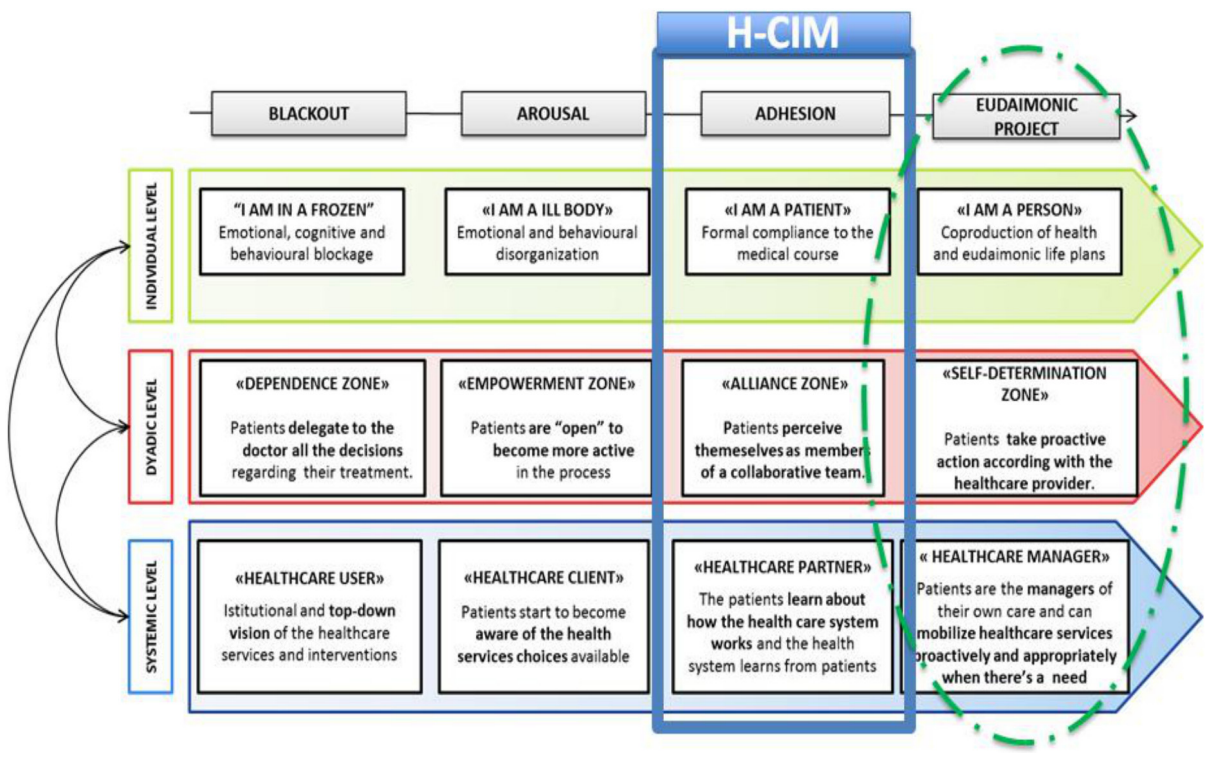

Figue 4: Implications of the PHE model for the H-CIM technology.

\section{Conclusion}

In the present chapter, we described a prototypical evaluation of a new technologybased intervention, from the joint viewpoint of user experience and patient engagement. According to this methodological approach, both usability measures and qualitative instruments can be employed to understand not only if the technology works as it should from a technical viewpoint, but also (1) if it is effectively understood and used by the patients and (2) appears consistent with their engagement level. We showed that the PHE model and the PHE questions may be helpful for clinicians when having to assess the effectiveness of a technology-mediated rehabilitation program and its attunement with patient engagement expectations.

\section{References}

Barello, S., Graffigna, G., \& Vegni, E. (2012). Patient Engagement as an Emerging Challenge for Healthcare Services: Mapping the Literature. Nursing Research and Practice, 2012, 1-7.

Brooke, J. (1996). SUS - A quick and dirty usability scale. In Usability Evaluation in Industry (pp. 189-194).

Forlizzi, J., DiSalvo, C., \& Gemperle, F. (2004). Assistive Robotics and an Ecology of Elders Living Independently in Their Homes. Human-Computer Interaction. 
Fuhrer, M.J. (2007). Assessing the efficacy, effectiveness, and cost-effectiveness of assistive technology interventions for enhancing mobility. Disability and Rehabilitation. Assistive Technology, 2, 149-158.

Graffigna, G., Barello, S., Wiederhold, B.K., Bosio, a C., \& Riva, G. (2013). Positive technology as a driver for health engagement. Studies in Health Technology and Informatics, 191, 9-17.

Gruman, J., Rovner, M.H., French, M.E., Jeffress, D., Sofaer, S., Shaller, D., \& Prager, D.J. (2010). From patient education to patient engagement: Implications for the field of patient education. Patient Education and Counseling, 78, 350-356.

Hart, S. ., \& Staveland, L.E. (1988). Development of NASA-TLX (Task Load Index): Results of empirical and theoretical research. In A. Hancock \& N. Meshkati (Eds.), Human Mental Workload. North Holland Press.

Hibbard, J.H., Stockard, J., Mahoney, E.R., \& Tusler, M. (2004). Development of the Patient Activation Measure (PAM): conceptualizing and measuring activation in patients and consumers. Health Services Research, 39, 1005-1026.

Huete, A.J., Victores, J.G., Martinez, S., Gimenez, A., \& Balaguer, C. (2012). Personal Autonomy Rehabilitation in Home Environments by a Portable Assistive Robot. IEEE Transactions on Systems, Man, and Cybernetics, Part C (Applications and Reviews), 42, 561-570.

Jette, D.U., Latham, N.K., Smout, R.J., Gassaway, J., Slavin, M.D., \& Horn, S.D. (2005). Physical therapy interventions for patients with stroke in inpatient rehabilitation facilities. Physical Therapy, 85, 238-248.

Kintsch, A., \& DePaula, R. (2002). A framework for the adoption of assistive technology. Learning Through Assistive Technology, 1-11.

Kousidou, S., Tsagarakis, N., Caldwell, D.G., \& Smith, C. (2006). Assistive Exoskeleton for Task Based Physiotherapy in 3-Dimensional Space. The First IEEE/RAS-EMBS International Conference on Biomedical Robotics and Biomechatronics. BioRob.

Laver, K., Ratcliffe, J., George, S., Burgess, L., \& Crotty, M. (2011). Is the Nintendo Wii Fit really acceptable to older people? A discrete choice experiment. 11:64 , BMC Geriatrics.

Lund, H.H. (2009). Modular robotics for playful physiotherapy. IEEE International Conference on Rehabilitation Robotics.

Maggiorini, D., Ripamonti, L.A., \& Zanon, E. (2012). Supporting seniors rehabilitation through videogame technology: A distributed approach. Second International Workshop on Games and Software Engineering: Realizing User Engagement with Game Engineering Techniques (GAS), 16-22.

Persad, C.C., Cook, S., \& Giordani, B. (2010). Assessing falls in the elderly: should we use simple screening tests or a comprehensive fall risk evaluation?. European Journal of Physical and Rehabilitation Medicine, 46, 249-259.

Riva, G., Baños, R.M., Botella, C., Wiederhold, B.K., \& Gaggioli, A. (2012). Positive technology: using interactive technologies to promote positive functioning. Cyberpsychology, Behavior and Social Networking, 15(2), 69-77.

Rizzo, A. “Skip”, \& Kim, G.J. (2005). A SWOT Analysis of the Field of Virtual Reality Rehabilitation and Therapy. Presence: Teleoperators and Virtual Environments, 14(2), 119-146.

Scherer, M. (2002). Assistive technology: Matching device and consumer for successful rehabilitation. Washington DC: American Psychological Association.

Smith, S.T., \& Schoene, D. (2012). The use of exercise-based videogames for training and rehabilitation of physical function in older adults: current practice and guidelines for future research. Aging Health, 8(3), 243-252.

Spielberger, C.D., Gorsuch, R.L., Lushene, P.R., Vagg, P.R., \& Jacobs, A.G. (1983). Manual for the State-Trait Anxiety Inventory (Form Y). Manual for the statetrait anxiety inventory STAI. Palo Alto, CA: Consulting Psychologists Press, (pp. 4-6). 
Tousignant, M., Boissy, P., Moffet, H., Corriveau, H., Cabana, F., Marquis, F., \& Simard, J. (2011). Patients' satisfaction of healthcare services and perception with in-home telerehabilitation and physiotherapists' satisfaction toward technology for post-knee arthroplasty: an embedded study in a randomized trial. Telemedicine Journal and E-Health : The Official Journal of the American Telemedicine Association, 17, 376-382. 